\title{
RIGOROUS QUANTUM FIELD THEORY MODELS
}

\author{
BY J. GLIMM ${ }^{1}$ AND A. JAFFE ${ }^{2}$
}

Communicated by M. H. Protter, October 20, 1969

Quantum field theory provides a natural framework to describe the interactions between elementary particles. Aside from a program of field theory computations, there has been an interest in qualitative or structural aspects of quantum field theory. We announce here some new progress in this direction. We study two standard nonlinear interactions in two space time dimensions. Our main results include the mathematically rigorous construction of quantum field theory models for these interactions.

We consider the interactions $\phi^{4}$ (a nonlinear self-coupling of mesons) and $\phi \bar{\psi} \psi$ (e.g. the coupling between mesons and neutrons or protons). In the nonrelativistic limit the $\phi \bar{\psi} \psi$ interaction gives rise to a Yukawa potential between fermions; thus it is called the Yukawa interaction.

The field equation for the $\phi^{4}$ theory is

$$
\phi_{t t}-\phi_{x x}+m^{2} \phi+4 \lambda \phi^{3}=0, \quad \lambda>0 .
$$

This equation was solved in [3] with the cube suitably defined. The field $\phi=\phi(x, t)$ of $(1)$ is a bilinear form, densely defined on a Hilbert space. The value of the bilinear form is continuous in $x$ and $t$, and the derivatives in (1) are distribution derivatives. The field is local

$$
[\phi(x, t), \phi(y, s)]=0, \quad \text { if }|x-y|>|t-s|,
$$

and satisfies the canonical commutation relations

$$
\left[\phi(x, t), \phi_{t}(y, t)\right]=i \delta(x-y) .
$$

The solution to (1) is obtained by solving the equation

$$
\phi_{t t}-\phi_{x x}+m^{2} \phi+4 \lambda g(x) \phi^{3}=0,
$$

AMS Subject Classifications. Primary 8135, 8146, 8147.

Key Words and Phrases. Quantum field theory models, Yukawa interaction, Hamiltonian, vacuum vector, Wightman axioms, Haag-Kastler axioms, locally Fock.

${ }^{1}$ Supported in part by the Air Force Office of Scientific Research under contract AF-49(638)1719.

2 Alfred P. Sloan Foundation Fellow. Supported in part by the Air Force Office of Scientific Research, and by a Shell grant at the Courant Institute of Mathematical Sciences 
where $g(x)$ is a nonnegative $C_{0}^{\infty}$ function. Equation (4) has a Hamiltonian

$$
H(g)=H_{0}+H_{I}(g),
$$

where $H_{0}$ is the Hamiltonian for $g \equiv 0$. The operator $H(g)$ is selfadjoint $[1]-[2]$, and in [3] it is proved that

$$
\phi_{\theta}(x, t)=e^{i t H(g)} \phi(x, 0) e^{-i t H(\theta)}
$$

is a solution to (3)-(4). Let $B$ denote a bounded interval and let $B_{t}$ be the set of all points within distance $|t|$ of $B$. If $x \in B$ and $g(x)=1$ on $B_{t}$, then $\phi_{\theta}(x, t)$ is a solution to (1), (3). A result of Segal [5, Theorem 3] based on the Trotter product formula ensures that such a $\phi_{o}(x, t)$ is independent of $g(\cdot)$ and that (2) is valid.

Our new results for the $\phi^{4}$ interaction concern the limit $g(\cdot) \rightarrow 1$.

Definition 1 . The selfadjoint operators $H$ and $P$ are energy and momentum operators for $\phi$ provided

$$
\begin{aligned}
e^{i t H} \phi(x, s) e^{-i t H} & =\phi(x, s+t), \\
e^{-i a P} \phi(x, s) e^{i a P} & =\phi(x+a, s), \\
H P & =P H .
\end{aligned}
$$

Definition 2. The unit vector $\Omega$ is a vacuum for $H, P$ if

$$
\begin{aligned}
& 0=H \Omega=P \Omega, \\
& 0 \leqq H .
\end{aligned}
$$

TheOREM 1. For the $\phi^{4}$ interaction in two dimensions there is a solution $\phi$ of (1)-(3) that has energy and momentum operators $H$ and $P$ and a vacuum vector $\Omega$. In other words $\phi, H, P$ and $\Omega$ satisfy (1)-(3) and (7)-(11).

It is known that the locally correct Hamiltonian $H(g)$ has a unique ground state vector $\Omega_{g}$ [3]. But $[H(g), P] \neq 0$ and $P \Omega_{g} \neq 0$, since $g$ and $H(g)$ are obviously not translation invariant. The $H$ and $\Omega$ of Theorem 1 are constructed as limits of $H(g)$ and $\Omega_{g}$ when $g(\cdot) \rightarrow 1$.

Define the $C^{*}$ algebra $\mathfrak{A}$ of bounded functions of the field as follows: Let $\mathfrak{A}(B)$ be the von Neumann algebra generated by

$$
\exp \left(i \int \phi(x, 0) f(x) d x\right), \quad \exp \left(i \int \phi_{t}(x, 0) f(x) d x\right)
$$

where $f=\bar{f} \in C_{0}^{\infty}(B)$ and $B$ is a bounded set. The algebra $\mathfrak{A}$ is the norm closure of $\bigcup_{B} \mathfrak{A}(B)$. 
The vector $\Omega_{g}$ determines a state $\omega_{g}$ in the Banach space dual $\mathfrak{I}^{*}$ of $\mathfrak{A}$,

$$
\omega_{\vartheta}(A)=\left(\Omega_{g}, A \Omega_{g}\right), \quad A \in \mathfrak{A} .
$$

After space averaging, we prove that a subsequence of the $\omega_{g}$ converge $w^{*}$ to a limit $\omega$. (Since $\mathfrak{A}$ is not norm separable, standard compactness arguments give only a convergent subnet.) We use $\omega \in \mathfrak{H}^{*}$ to define the inner product in a new Hilbert space $\mathfrak{H C}$ (the Gelfand-Segal construction). We have a representation $\pi$ of $\mathfrak{A}$ as operators on $\mathfrak{H C}$ such that

$$
\omega(A)=(\Omega, \pi(A) \Omega)
$$

for some $\Omega \in \mathcal{F}$.

Definition 3. A representation $\pi$ of $\mathfrak{A}$ as operators on a Hilbert space is called locally Fock if for each $\mathfrak{A}(B)$,

$$
\pi \uparrow \mathfrak{A}(B)
$$

is unitarily equivalent to the identity representation.

THEOREM 2. The representation $\pi$ of (13) is locally Fock.

We use this theorem to construct $H$ and $P$. It also allows us to extend the representation $\pi$ both to the unbounded operators $\int \phi(x, t) f(x, t) d x d t$ and to the bilinear form $\phi(x, t)$. Our $\phi^{4}$ field theory satisfies most of the Haag-Kastler axioms and many of the Wightman axioms.

We now turn to the Yukawa interaction in two dimensions; we call it $Y_{2}$ for short.

THEOREM 3. For the $Y_{2}$ field theory, the space cutoff energy operator $H(g)$ is selfadjoint.

We observe that $H(g)$ is a remarkably singular operator, because its formal expression

$$
H_{0}+H_{I}(g)-\frac{1}{2} \delta m^{2} \int \phi^{2} g(x)^{2} d x-E_{0}
$$

contains terms with infinite coefficients [4], namely $\delta m^{2}$ and $E_{0}$. (The Hamiltonian $H$ for the $\left(\phi^{4}\right)_{2}$ theory also contains an infinite constant.) We define $H(g)$ as the limit of a sequence of approximate, selfadjoint Hamiltonians. The proof of the existence of an operator $H(g)$ involves the explicit construction of vectors $\theta$ in the domain of $H(g)$. In the formal expression for $H(g) \theta$, there are a number of infinite terms that cancel. We prove Theorem 3 for this limit. 
We have established the finite propagation speed and locality for the $Y_{2}$ theory. As before, we define $\mathfrak{A}$ to be the $C^{*}$ algebra generated by bounded functions of the time zero fields.

TheOREM 4. In the $Y_{2}$ theory, let $A \in \mathfrak{A}(B)$ and $g(x)=1$ on $B_{t}$. Then

$$
\sigma_{t}(A)=e^{i t H(o)} A e^{-i t H(o)} \in \mathfrak{A}\left(B_{t}\right),
$$

and $\sigma_{t}(A)$ is independent of $g(\cdot)$.

Here $\sigma_{t}$ defined by (15) is a one parameter group of time translation automorphisms of $\mathfrak{A}$. Using $\sigma_{t}$ we also construct canonical fields $\phi(x, t)$ and $\psi(x, t)$ which commute or anticommute at space-like separated points. Most of the Haag-Kastler axioms are satisfied for our $Y_{2}$ quantum field theory.

Because of the infinite counterterms in $H(g)$, Theorem 4 does not follow from the Trotter product formula.

\section{REFERENCES}

1. J. Glimm and A. Jaffe, Singular perturbations of self adjoint operators, Comm. Pure Appl. Math. 22 (1969), 401-414.

2. - $A \lambda \phi^{4}$ quantum field theory without cutoffs. I, Phys. Rev. 176 (1968), 1945-1951.

3. - The $\lambda\left(\phi^{4}\right)_{2}$ quantum field theory without cutoffs. II: The field operators and the approximate vacuum, Ann. of Math. (to appear).

4. - An infinite renormalization of the Hamiltonian is necessary, J. Math. Phys. (to appear).

5. I. Segal, Notes toward the construction of nonlinear relativistic quantum fields. I: The Hamiltonian in two space-time dimensions as the generator of a $C^{*}$-automorphism group, Proc. Nat. Acad. Sci. U.S.A. 57 (1967), 1178-1183. MR 35 \#5195.

Courant Institute of Mathematical Sciences, New York University, New York, New York 10012 aND

Lyman Laboratory of Physics, Harvard University, Cambridge, MassaChUSETTS 02138 